\title{
Maize rhm1 Resistance to Bipolaris maydis Is Associated with Few Differences in Pathogenesis-Related Proteins and Global mRNA Profiles
}

\author{
Carl R. Simmons, ${ }^{1}$ Susan Grant, ${ }^{1}$ Daniel J. Altier, ${ }^{1}$ Patrick F. Dowd, ${ }^{2}$ Oswald Crasta, ${ }^{3}$ Otto Folkerts, ${ }^{3}$ \\ and Nasser Yalpani ${ }^{1}$ \\ ${ }^{1}$ Pioneer Hi-Bred International, 7300 N.W. 62nd Avenue, Johnston, IA 50131-1004, U.S.A.; ${ }^{2}$ USDA-ARS, \\ 1815 N. University Street, Peoria, IL 61604-3999, U.S.A.; ${ }^{3}$ CuraGen, 555 Long Wharf Drive, New Haven, \\ CT 06511, U.S.A. \\ Submitted 1 November 2000; Accepted 30 March 2001.
}

The maize rhm1 mutant resists Bipolaris maydis, the causal agent of Southern corn leaf blight, by producing small necrotic lesions surrounded by chlorotic haloes. The rhm1 and wild-type lesions contain viable fungus in equal frequency, but fungal sporulation was markedly inhibited on rhm1. The levels of the pathogenesis-related (PR) proteins chitinase, PR1, and peroxidase differ little between rhm1 and wild type, with or without $B$. maydis inoculation. The global mRNA profiles surveyed revealed hundreds of cDNA fragments that were twofold or more induced or suppressed in rhm1 and wild-type plants following $B$. maydis inoculation. Nonetheless, between $\mathrm{rhml}$ and wild type, only 0.4 to $0.7 \%$ of the cDNA fragments were expressed differentially by twofold or more. Among the upregulated genes in $\mathrm{rhm} 1$ was beta-glucosidase glu1, which prompted a test of whether $\mathrm{rhm} 1$ resistance depends upon the antimicrobial compound 2,4-dihydroxy-7-methoxy-1,4benzoxazin-3-one or other hydroxamic acids whose glucosyl conjugates are preferred substrates for the Glu1 enzyme. Double mutants of rhml and bxi, a hydroxamic acid-deficient mutant, indicate that rhm 1 resistance is hydroxamic acid independent. The $r h m 1$ resistance presently appears to operate via a mechanism unlike those of previously described resistance genes.

Additional keywords: Cochliobolus heterostrophus, Helminthosporium maydis.

Bipolaris maydis is the fungal pathogen responsible for Southern corn leaf blight, which typically causes tan, elliptical-to-rectangular leaf lesions (White 1999). B. maydis, previously known as Helminthosporium maydis, is the anamorph form of the ascomycete teleomorph Cochliobolus heterostrophus. It exists principally as race $\mathrm{O}$ and, to a lesser extent, as race T. Maize cultivars are often susceptible to $B$. maydis and, in the early $1970 \mathrm{~s}, B$. maydis race $\mathrm{T}$ caused an epidemic on Texas male sterile cytoplasm, de-

Corresponding author: C. Simmons; Telephone: +1-515-270-5949; Fax: +1-515-270-4778; E-mail: simmonscr@phibred.com stroying nearly $15 \%$ of the U.S. corn crop (Smith and White 1988).

There are various sources of genetic resistance to $B$. maydis, some oligogenic, others polygenic (Burnette and White 1985; Holley and Goodman 1989; Smith and White 1988). A monogenic recessive resistance mechanism was discovered in Nigerian breeding stock in the late 1960s. This resistance is characterized by small necrotic spots surrounded by chlorotic haloes and little fungal sporulation and is expressed in young plants prior to anthesis (Craig and Fajemisin 1969). The genetic locus was named rhml for resistance to Helminthosporium maydis (Smith and Hooker 1973) and, subsequently, was mapped to the short arm of chromosome 6 near the rgdl locus and the restriction fragment length polymorphism marker umc85 (Neuffer et al. 1997; Zaitlin et al. 1993). Little has been reported about the molecular physiology of rhml resistance. Increased levels of secondary metabolites such as phenolics, however, are associated with other maize resistance mechanisms to B. maydis (Sharma et al. 1983).

Plants defend themselves through a variety of mechanisms, among them cell wall structural alterations, creating barriers to infection, antimicrobial metabolites, and antimicrobial protein expression (Agrios 1997). Sometimes resistance is accompanied by the expression of diverse defense-related genes, many of which are referred to as pathogenesis-related (PR) proteins and some with known antimicrobial activity (Cutt and Klessig 1992; Linthorst 1991). Frequently, in resistant reactions such as gene-for-gene hypersensitive responses (HR) governed by $\mathrm{R}$ genes, the induction of $\mathrm{PR}$ proteins is more rapid and proceeds to higher levels, at least early after infection (Esnault et al. 1993; Vad et al. 1993). Additionally, constitutive resistance often occurs in association with the overexpression of defense-related genes (Chen et al. 1999; Clarke et al. 1998; Nishizawa et al. 1999). As a result, a comparison of gene expression patterns, in particular that of $\mathrm{PR}$ protein genes in resistant versus susceptible cultivars, can provide clues to the molecular mechanisms of resistance.

Studies of PR protein expression typically rely on the detection of one or a small number of proteins or genes. Recent advances in gene sequence databases and genomics technology, however, provide new opportunities to globally investi- 
gate thousands of gene expression changes. Among such genomic-scale profiling technologies are those based on mRNA differential display (Shimkets et al. 1999). In this study, we profile PR protein accumulation and global mRNA levels in rhml and wild-type plants in an effort to better understand the rhml resistance mechanism. We also present evidence that the rhml resistance to $B$. maydis involves an apparently novel mechanism, one associated with few PR protein and mRNA expression differences relative to the total number of postinoculation defense-related expression changes.

\section{RESULTS}

\section{B. maydis resistance in $\mathrm{rhm} 1$.}

Three maize plants resistant to $B$. maydis were recovered from a screen of a population containing active Robertson Mutator transposons. The resistance was characterized by small chlorotic lesions, similar to rhml. Subsequent crosses confirmed that they were allelic to each other and to the published $r h m l$ allele (here named $r h m l-\mathrm{R}$ ). For this study, we named these new alleles $r h m l-\mathrm{A}, r h m l \mathrm{~B}$, and $r h m 1-\mathrm{C}$. Sideby-side comparisons and phenotypic scoring in $\mathrm{F}_{2}$ and backcross populations indicated that the alleles varied slightly in strength (by lesion size), with rhml-R the strongest, followed by rhml-A, rhml-B, and rhml-C. It is possible, however, that these are not allelic differences but rather genetic background effects. The rhml-A line exhibited some spontaneous lesions, although $\mathrm{F}_{2}$ analysis indicated that the lesion phenotype was semidominant and did not cosegregate with the rhml phenotype. Nonetheless, as a result of the lesion phenotype, rhml-A was not used for mRNA profiling. The B. maydis-infected

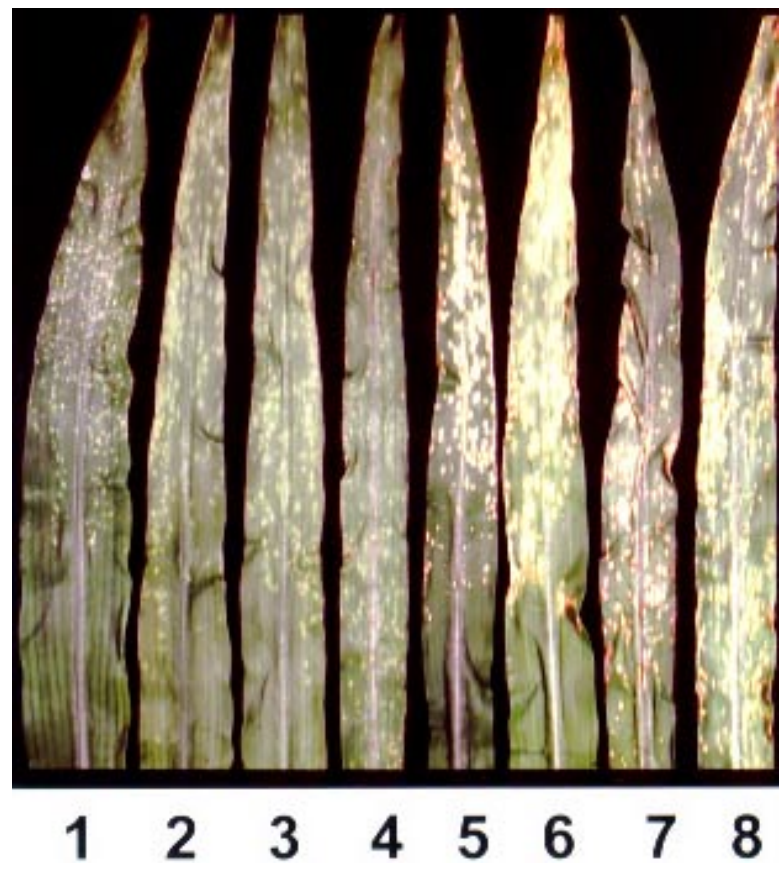

Fig. 1. Wild-type and $r h m 1$ leaves 3 days postinoculation with Bipolaris maydis. Note the reduced lesion size on $r h m 1$ plants. 1, rhml-R (co-op); 2, rhml-A; 3, rhml-B; 4, rhm1-C; 5, rhm1-C-PHAAO wild-type heterozygote; 6 , rhml-C-PHAAO $\mathrm{F}_{2}$ wild-type homozygote; 7, B73; 8, PHAAO. The visible rhml-A lesions are the result of infection and not the mimicry sometimes exhibited by this line. leaves of the four rhml alleles and various wild-type susceptible lines are presented in Figure 1.

The rhml and wild-type lesions were assayed for whether they contain viable $B$. maydis fungus by plating surfacesterilized leaf tissue containing a lesion margin onto agar medium. When the fungus was present, it formed an outgrowth or propagule onto the agar, and the lesion was considered "fertile." The four rhml alleles were compared with their wildtype susceptible counterparts and two other wild-type lines with the use of an average of 123 lesions per genotype. The four $r h m 1$ alleles mostly had fertile lesions, with an average of $82.4 \%$ (standard error $[\mathrm{SE}]=4.0 \%$ ) of the lesions yielding propagules. The four corresponding wild types averaged $84.1 \%$ ( $\mathrm{SE}=9.0 \%)$, whereas the two other wild-type lines averaged $90.1 \%(\mathrm{SE}=1.5 \%)$. This analysis therefore indicates that viable fungus was present in the rhml and wild-type lesions at similar frequencies.

When $B$. maydis-infected wild-type leaves were surface sterilized and transferred to a humid chamber for $40 \mathrm{~h}, B$. maydis conidia erupted on the leaf surface, within and outside the lesions (Fig. 2A). Infected rhml leaves of all four alleles displayed few conidia, however, either within or between the lesions. Some rhml lesions had no conidia, whereas others had tufts of conidia, usually only in the lesion area (Fig. 2B and $\mathrm{C}$ ). If the conidia were left in the humid chamber for several additional days, it would eventually become more common on the rhml leaf surfaces, although their appearance was markedly delayed relative to wild type. In addition, the conidia tended to be smaller on rhml. This result indicates that rhml retards in vivo B. maydis sporulation.

\section{Pathogenesis-related (PR) protein accumulation.}

The accumulation of PR 1 and chitinase in rhml and wild type were monitored following $B$. maydis inoculation with Western blots (Fig. 3). rhml and wild type were observed to have substantially increased accumulation of PR1 and chitinase protein following inoculation. Their accumulation was scarcely detectable at $0 \mathrm{~h}$ postinoculation (hpi). By $16 \mathrm{hpi}$, however, their accumulation was elevated, with levels peaking at approximately $48 \mathrm{hpi}$. PR1 accumulation levels rose to similar levels in rhml and wild type, although in $\mathrm{rhml}$, the accumulation lagged slightly behind wild type at 16 and 24 hpi. For chitinase, the timing and level of accumulation was similar between rhml and wild type. Peroxidase expression analyzed by isoelectric-focusing activity gels in all four rhm l alleles, with or without $B$. maydis inoculation, indicates that even though several anionic peroxidase species are defense induced, there is no consistent difference between rhml and wild types in the level or species of peroxidase isozyme being expressed (not shown).

\section{Global mRNA profiles are similar between $r h m 1$ and wild type.}

CuraGen GeneCalling differential display technology (New Haven, CT, U.S.A.) was used to survey global mRNA expression changes in uninfected or B. maydis-infected rhml and wild-type plants. The single 24 -h time point was chosen because at that time rhml and wild-type lesions were approximately the same size. Consequently, it was reasoned that their mRNA expression differences would more likely be related to the resistance mechanism and not to the secondary effects 
caused by diverged symptom development. Three independent experiments were performed: two comparing rhml-C with wildtype $\mathrm{C}$, the third comparing rhml-B with wild-type $\mathrm{B}$. The number of cDNA fragments detected ranged from 8,125 to 13,724 (Table 1). Thousands of genes were thus assayed, although somewhat fewer genes than the number of cDNA fragments assayed because some cDNA fragments may have derived from the same gene. Nonetheless, these genes represent a large sampling that are unbiased as to function.

rhml and wild-type plants responded to B. maydis inoculation through changes in mRNA expression. For the purposes of this study, a change in expression generally means a mini-
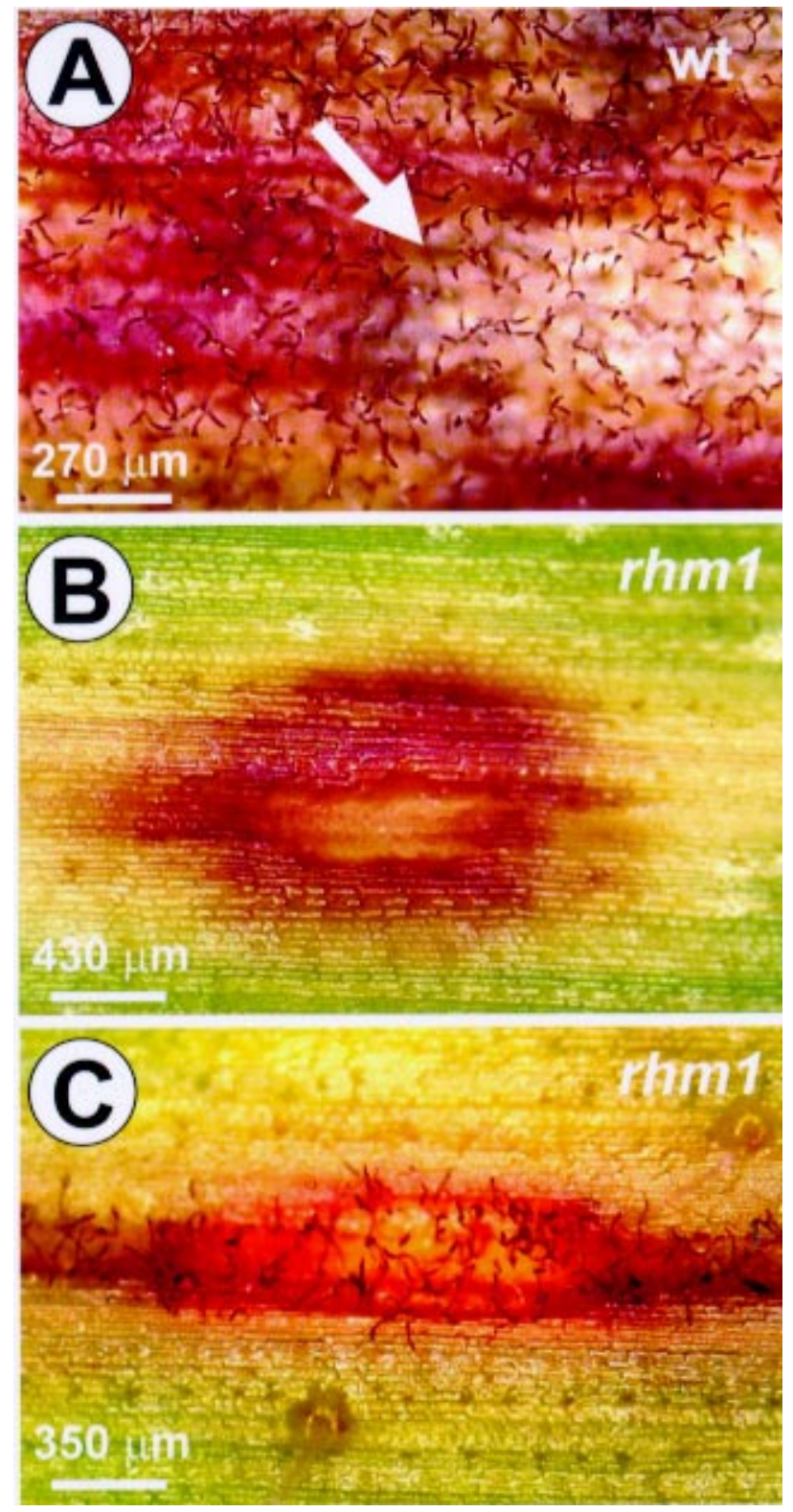

Fig. 2. The rhml lesions exhibit reduced Bipolaris maydis sporulation. $\mathbf{A}$, Wild-type and $\mathbf{B}$ and $\mathbf{C}, \mathrm{rhm} 1$ leaves were inoculated with B. maydis and, after 3 days, stored in a humid chamber for $40 \mathrm{~h}$ to induce conidia formation. Note that on the wild-type leaves, conidia are profuse within and outside the lesions (lesion margin is reddish, as indicated by the arrow in $\mathbf{A}$ ), whereas in rhml lesions, the conidia are absent (B) or fewer and more confined to the lesion $(\mathbf{C})$. mum of a twofold change in abundance upward or downward. In experiment $1,17.9$ to $19.0 \%$ of the cDNA fragments changed in expression, with approximately $65 \%$ being up regulated. In experiments 2 and 3, the percentage of cDNA fragments that changed in expression was lower, approximately 4 and $6 \%$, respectively, but the proportion that was up regulated remained at similar levels, 62 and $74 \%$ (Table 1). The lower percentage expression differences in experiments 2 and 3 apparently are the result of these experiments being less advanced physiologically in the defense response.

In order to make comparisons within and between experiments, sets of cDNA fragments that showed a difference in gene expression between $r h m 1$ and wild type across all three experiments were identified. The overall global average fold change of these cDNA fragments for each experiment is presented in Table 2 . For experiment 1 , the up-regulated cDNA fragments averaged an approximate fourfold increase, whereas the down-regulated bands averaged a threefold decrease. For experiments 2 and 3, the average increase or decrease was lower, which is consistent with these experiments being less advanced physiologically in the defense response. What is notable about these comparisons, however, is that regardless of the experiment and the number of common cDNA fragments used for calculation, the average difference in upward or downward expression between rhml and wild type was nearly the same.

In addition to calculating the overall average fold change, a ratio of the fold changes for each of the differentially expressed common cDNA fragments between rhml and wild type was calculated independently. Afterward, the ratios were averaged across all the common cDNA fragments used for the calculation (Table 2). This, too, affirmed that there was little relative change in mRNA expression between rhml and wild type in these three separate profiling experiments. The ratios were very near to 1.00 and, in the largest study involving 770 up-regulated cDNA fragments, the ratio was $1.00(\mathrm{SE}=0.02)$. In short, in these three separate experiments, there was not a significant difference in the level of mRNA expression between $r h m l$ and wild type after $B$. maydis inoculation. Moreover, these three experiments differed somewhat in their physiological response to the inoculation, which indicates that the lack of global difference between rhml and wild-type defense-related mRNA expressions was not limited to one strict physiological point following inoculation.

\section{rhm1 | Wildtype}

$\begin{array}{lllllllllllll}1 & 2 & 3 & 4 & 5 & 6 & 1 & 2 & 3 & 4 & 5 & 6 & \mathrm{C}\end{array}$

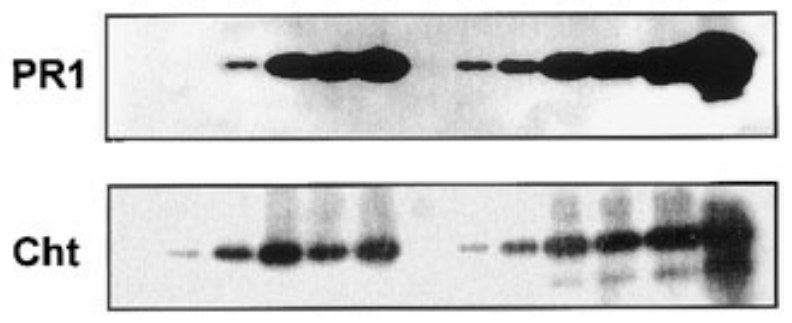

Fig. 3. Western analysis of PR1 and chitinase (Cht) expression in rhml and wild-type leaves following infection with Bipolaris maydis. Tissue was sampled at 0 (1), 16 (2), 24 (3), 48 (4), 72 (5), and 96 (6) h. A positive control for PR1 and chitinase, migrating at 16 and $30 \mathrm{kDa}$, respectively, is in lane $\mathrm{C}$. 
Differences in mRNA expression between $r h m 1$ and wild type.

We observed few gene expression differences between rhml and wild type in each of three mRNA profiling experiments (Table 3), regardless of whether it was an uninoculated or inoculated comparison. Only 0.4 to $0.7 \%$ of the cDNA fragments were expressed differentially in each comparison. Summed across all experiments, 187 cDNA fragments were up regulated and 191 were down regulated. No differentially expressed cDNA fragment was common to all three experiments. Between experiments 2 and 3, there were five cDNA fragments in common with the uninoculated or inoculated rhml-to-wild-type comparisons of both experiments, four of which were down regulated in rhml. Three of these downregulated fragments were confirmed by competitive polymerase chain reaction (PCR): two corresponded to phosphoenolpyruvate (PEP) carboxylase (accession no. P04711), and one to the chlorophyll A B binding protein (CAB) (accession no. P08221). Among the inoculated rhml-to-wild-type comparisons of experiments 2 and 3, there were 25 common cDNA fragments, 20 of which were down regulated. For the uninoculated controls, there were 13 common cDNA fragments, six that were up regulated and seven that were down regulated.

Of the 89 cDNA fragments that were at least twofold higher expressed in rhml versus wild type after inoculation in experiment 1 (Table 2), six matched the eight predicted cDNA fragments for the Zea mays beta-glucosidase glul cDNA sequence (GenBank accession no. X74217), giving glul the most significant GeneCall score in the entire experiment. The seventh cDNA fragment had a fold change of 1.74. An unrelated comigrating band interfered with analysis of the eighth. The average fold change of all seven glul bands was 2.93 out of a 1.74 to 4.27 range $(\mathrm{SE}=0.35$ ). The glul origination of two such bands was confirmed by competitive PCR (see below), which specifically eliminated the glul bands, leaving the neighboring bands on the gel trace unaffected (data not shown).

The maize glul (accession nos. X74217 or U25157) and glu2 (accession no. U44087) genes are closely related, having 90\% nucleotide identity (GAP program from the Wisconsin Sequence Analysis software package; Genetics Computer Group, Madison, WI, U.S.A.). The investigation of over 400,000 maize cDNAs at Pioneer Hi-Bred International (Johnson, IA, U.S.A.) revealed no other gene that was more than $70 \%$ identical to the nucleotide sequence of glul or glu2. Because glul and glu2 were closely related, they shared some cDNA fragments. The two up-regulated cDNA fragments confirmed by competitive PCR, however, were unique to glul. The average fold change of the five cDNA fragments that were unique to glu 2 was only 1.09 ( $\mathrm{SE}=0.14)$. Thus, this affirmed that glul, not glu2, was observed to be up regulated in $r h m 1$ relative to wild type. Northern blot analysis also indicated that following inoculation, glul mRNA expression was higher in $r h m l-R$ relative to wild type (data not shown). In uninoculated control plants from experiment 1 , there was only a slight difference in glul expression between $\mathrm{rhml}$ and wild type: the glul cDNA fragments averaged 1.29-fold ( $\mathrm{SE}=$ $0.16)$, and the glu2 fragments averaged 1.00 -fold ( $\mathrm{SE}=0.04)$.

Independent of the difference between rhm 1 and wild type, experiment 1 profiling data indicates that glul and glu 2 mRNA expression increased following inoculation, although the increase was greater for glul. For the glul cDNA fragments, the average increase was 9.7 -fold out of a 2.41 to 21.75 range $(\mathrm{SE}=4.85)$ and, for glu2-unique cDNA fragments, the

Table 1. Global mRNA expression profiles of $r h m 1$ and wild type following Bipolaris maydis inoculation

\begin{tabular}{|c|c|c|c|c|c|c|}
\hline & \multicolumn{2}{|c|}{ Experiment 1} & \multicolumn{2}{|c|}{ Experiment 2} & \multicolumn{2}{|c|}{ Experiment 3} \\
\hline & rhm1-C & Wild-type-C & rhm1-C & Wild-type-C & rhm1-B & Wild-type-B \\
\hline Total cDNA fragments assayed & 11,523 & 13,291 & 13,591 & 13,075 & 13,724 & 13,459 \\
\hline cDNA fragments \pm twofold ${ }^{\mathrm{a}}$ & 2,185 & 2,375 & 458 & 524 & 835 & 814 \\
\hline Percentage of total assayed & 19.0 & 17.9 & 3.4 & 4.0 & 6.1 & 6.1 \\
\hline Percentage up regulated & 64.1 & 65.7 & 62.0 & 68.3 & 74.4 & 73.5 \\
\hline
\end{tabular}

${ }^{a}$ At least twofold differentially expressed in a comparison between inoculated and uninoculated controls.

Table 2. Global mRNA expression profiles for $r h m 1$ and wild type following Bipolaris maydis inoculation. Global average fold change of cDNA fragments, and average $r h m l$ versus wild-type ratio per cDNA fragment ${ }^{\mathrm{a}, \mathrm{c}}$

\begin{tabular}{|c|c|c|c|c|c|c|c|c|c|}
\hline \multirow[b]{2}{*}{ Number $^{\text {b }}$} & \multicolumn{3}{|c|}{ Experiment 1} & \multicolumn{3}{|c|}{ Experiment 2} & \multicolumn{3}{|c|}{ Experiment 3} \\
\hline & rhm1-C & Ratio $^{c}$ & Wild-type C & rhm1-C & Ratio $^{c}$ & Wild-type C & rhm1-B & Ratio & Wild-type B \\
\hline \multicolumn{10}{|l|}{ Upward } \\
\hline 46 & $4.78^{\mathrm{a}}$ & 1.17 & $4.20^{\mathrm{a}}$ & 2.42 & 1.02 & 2.49 & 3.07 & 1.16 & 2.88 \\
\hline 126 & & & & 2.77 & 1.04 & 2.84 & 3.30 & 1.11 & 3.22 \\
\hline 770 & 4.00 & 1.00 & 3.76 & & & & & & \\
\hline \multicolumn{10}{|l|}{ Downward } \\
\hline $6^{\mathrm{d}}$ & 2.70 & 1.02 & 2.66 & 1.98 & 1.00 & 1.97 & 2.17 & 1.04 & 2.25 \\
\hline 35 & & & & 2.22 & 1.01 & 2.36 & 1.38 & 1.13 & 2.34 \\
\hline 369 & 3.89 & 1.13 & 3.76 & & & & & & \\
\hline
\end{tabular}

${ }^{a}$ Average fold changes for the combined sum of all two- to tenfold changed cDNA fragments for each genotype, comparing inoculated versus uninoculated controls.

${ }^{\mathrm{b}}$ Number of cDNA fragments used for the calculation, each with two- to tenfold shared changes in expression within and between experiments.

${ }^{\mathrm{c}}$ Within an experiment, the average of all the individual $r h m l$ versus wild-type fold change ratios for each common two- to tenfold changed cDNA fragments in common between rhml and wild type.

${ }^{\mathrm{d}}$ For the six common down-regulated cDNA fragments, the fold change was relaxed to 1.5 - to tenfold so that sufficient bands bridging all three experiments could be identified. 
increase was only 2.12 -fold out of a 1.23 to 4.17 range $(\mathrm{SE}=$ 0.70). This is believed to be the first study to report defenseinduced glul or glu 2 mRNA expression. In profiling experiments 2 and 3, the glul cDNA fragment expression was induced, at most, twofold and there was little, if any, difference in glul expression between $r h m l$ and wild type. These physiologically less-advanced experiments may have caught glul induction earlier when there was yet little difference in glul expression between $\mathrm{rhml}$ and wild type.

\section{The $r h m 1$ resistance is independent of hydroxamic acids.}

The increased beta-glucosidase glul expression profiles evoked the hypothesis that $r h m l$ resistance may be controlled by the liberation of free hydroxamic acids such as 2,4dihydroxy-7-methoxy-1,4-benzoxazin-3-one (DIMBOA) from generally less active glucosyl conjugates (Bravo and Niemeyer 1991; Queirolo et al. 1981). DIMBOA glucosides are a preferred substrate for Glu1, which releases the aglycone DIMBOA as product (Babcock and Esen 1994; Cuevas et al. 1992). Hydroxamic acids, in particular DIMBOA, are cerealspecific, seedling-associated antimicrobial metabolites, whose levels are correlated to maize resistance and fungi, including B. maydis (Niemeyer 1988). We observed higher unglycosylated DIMBOA levels in all four rhml alleles relative to wild type (not shown). As a result, we sought to directly test whether or not rhml (chromosome 6) depended upon DIMBOA by crossing it to the bxl mutation (chromosome 4), which disrupts hydroxamic acid production (Frey et al. 1997). For the $\operatorname{rhml}-B \mathrm{~F}_{2}$ population $(N=178$ of 220 planted $)$, the phenotypic ratio (Bxl-Rhml:Bxl-rhml:bxl-Rhml:bxl-rhml) was 9.9:2.2:3.4:0.5 $\left(X^{2}=29.4, p<0.005\right)$. For the $r h m 1-C \mathrm{~F}_{2}$ population $(N=166$ of 220 planted), the phenotypic ratio was 12.0:2.6 to 1.0:0.4 $\left(X^{2}=6.6,0.1>p>0.05\right)$. The ratios generally followed a 9:3:3:1 pattern for the assortment of two independent recessive phenotypes. These results clearly indicate that $r h m l$ resistance does not depend upon $B x 1$ gene function, hydroxamic acids, or DIMBOA. Furthermore, in $b x 1-$ mutant/Rhml-wild-type seedlings, we did not observe generally enhanced $B$. maydis susceptibility, suggesting that even apart from rhml, resistance to B. maydis is not controlled significantly by DIMBOA. Additionally, up to $10 \mathrm{mg}$ of DIMBOA per $\mathrm{ml}$ did not inhibit $B$. maydis growth or sporulation in agar plate radial diffusion assays (data not shown).

\section{DISCUSSION}

The primary purpose of this study was to gain understanding of the molecular basis of $r h m l$ resistance to B. maydis through profiling the expression of PR proteins and mRNAs. Between $r h m l$ and wild type, the defense-related proteins
PR1, chitinase, and peroxidases generally were found not to differ in their level or timing of expression, with or without inoculation by B. maydis. In rhml, the PR1 protein levels increased somewhat more slowly after inoculation with $B$. maydis. We interpret that this does not reflect the resistance mechanism itself, but rather indicates reduced tissue damage in resistant rhml tissue, resulting in less PR1 induction. Interestingly, in rhml there was no evidence of defense-induced cationic peroxidase, which often is associated with other resistant disease reactions in maize (Dowd 1994) and other cereals (Reimers et al. 1992).

Supporting the protein-profiling conclusion, three separate mRNA profiling experiments were performed, each assaying thousands of genes. These revealed that rhml and wild-type plants differed little in gene expression patterns, whether or not the plants were infected by $B$. maydis. After $B$. maydis inoculation, hundreds of cDNA fragments, two-thirds of which were induced, changed twofold or more in expression at $24 \mathrm{~h}$. Ongoing analysis indicates that many of these induced genes are familiar PR genes such as chitinases, but many others are unknown or novel genes. Of the nearly 30 confirmed genes that were induced by inoculation, all are clearly maize genes by virtue that they match maize expressed sequence tag (EST) sequences or other plant genes, indicating that cDNAs from $B$. maydis are not a significant profiling contaminant. Together with the PR protein analysis, mRNA profiling indicates that the rhml resistance mechanism does not involve a general constitutively activated or hyperinduced expression of defense-related genes.

Comparing rhml with wild type, we observed few mRNA expression changes that were common to the three mRNA profiling experiments. The majority were down expressed in rhml, either constitutively down or down only after inoculation, relative to wild type. These genes remain under investigation, but apart from glul, they do not appear to be defense related. We suspect that many of these gene expression changes reflect secondary byproducts of the resistance mechanism such as the down regulation of PEP carboxylase and $\mathrm{CAB}$ in rhml, which may relate to the chlorosis manifested in $r h m l$ lesions. These mRNAs point to a perturbation of the chloroplast or photosynthesis. In fact, the glul betaglucosidase is expressed in chloroplasts (Esen and Stetler 1993) but also is located outside organelles (Feldwisch et al. 1994). This suggests that at least for rhml, chloroplasts may somehow participate in the resistance reaction.

The observation in this study that beta-glucosidase glul was one of the few surveyed genes that was expressed differently in $r h m 1$, and is the only gene with an apparent potential role in defense, naturally focused attention on the glul gene. Because glul is believed to function in maize defense by releas-

Table 3. Differences in mRNA expression in rhml versus wild type, with or without Bipolaris maydis infection in three mRNA profiling experiments

\begin{tabular}{|c|c|c|c|c|c|c|}
\hline & \multicolumn{2}{|c|}{ Experiment $1^{\mathrm{a}}$} & \multicolumn{2}{|c|}{ Experiment $2^{a}$} & \multicolumn{2}{|c|}{ Experiment $3^{a}$} \\
\hline & Control & Infected & Control & Infected & Control & Infected \\
\hline Total cDNA fragments assayed & 8,125 & 11,884 & 13,453 & 13,242 & 13,526 & 13,632 \\
\hline Fragments with twofold difference ${ }^{b}$ & 30 & 89 & 76 & 62 & 66 & 65 \\
\hline Total fragments assayed & $0.4 \%$ & $0.7 \%$ & $0.6 \%$ & $0.5 \%$ & $0.5 \%$ & $0.5 \%$ \\
\hline Up-down expressed & $11-19$ & $54-35$ & $41-35$ & $32-30$ & $22-34$ & $27-38$ \\
\hline
\end{tabular}

${ }^{a}$ Experiments 1 and 2 involve rhm1-C versus wild-type C, and experiment 3 involves rhm1-B versus wild-type B.

$\mathrm{b}$ cDNA fragment expression differences that were changed at least twofold upward or downward in infected (inoculated) versus control (uninoculated). 
ing antimicrobial compounds, in particular DIMBOA, from their less active glucosyl conjugates, we directly tested whether rhml depended upon DIMBOA and hydroxamic acids by a genetic test involving $b x 1-r h m l$ double mutants. The rhml resistance was clearly shown by this test to operate independently of DIMBOA and other hydroxamic acids.

There are many other known plant antimicrobial or defense compounds, many of which exist as less active glycosides (Osbourn 1996). Similar to DIMBOA, some of these compounds are more prevalent in seedlings than in mature plants, mirroring the developmental profile of $\mathrm{rhml}$ resistance. There also are other substrates known for beta-glucosidase Glu1 (Babcock and Esen 1994; Esen 1993; Hughes 1993), although substrate ranges for beta-glucosidases, in general, are not well understood (Conn 1993). The fact that increased levels of secondary metabolites such as phenolics are associated with maize resistance to $B$. maydis adds intrigue (Sharma et al. 1983). Consequently, the hypothesis that rhml operates through beta-glucosidase Glu1 releasing increased amounts of antimicrobial aglycones presently remains attractive, particularly in light of the fact that few other gene expression changes were observed. There are presently no confirmed glul gene knockout mutations, or mutations such as $b x l$ which knock out other classes of antimicrobial compounds, through which a similar direct genetic test of this hypothesis can be performed.

Plants defend themselves by many mechanisms, although frequently through alterations in the level or timing of PR protein expression, as can occur in gene-for-gene HR responses. The protein and mRNA expression data from this study indicate that rhml involves few such gene expression changes. Nonetheless, it should be emphasized that there are many genes whose expression was not assayed in these experiments, which might relate to $r h m l$ resistance. From this study, it also is evident that rhml lesions contain viable fungus, although sporulation is inhibited, unlike many HR reactions that rapidly form generally sterile lesions. In addition, rhml resistance is recessive, unlike many R-gene triggered HR reactions. In some ways, rhml resembles the barley recessive, nonrace-specific resistance to Erisiphe graminis, which is controlled by the Mlol gene (Buschges 1997). The mlo resistance mechanism, however, is associated with increased expression of defense-related genes (Peterhaensel et al. 1997). Because rhml maps to chromosome 6, bin 1, close to genes related structurally to characterized $\mathrm{R}$ genes, led us to speculate that $r h m 1$ may be such a $\mathrm{R}$ gene. Our extensive analysis of several hundred Mutator transposable elements in each of the three rhml mutants described here did not identify any that were tightly linked to Rhml. Nevertheless, the glul gene is not the $R h m l$ gene because it is located on chromosome 10 (Pryor 1978), whereas Rhml is on chromosome 6. The Rhml gene structure thus remains unknown. Nonetheless, by the varied criteria mentioned above, the rhml resistance mechanism does not appear to fit existing $\mathrm{R}$ gene-HR resistance models.

\section{MATERIALS AND METHODS}

Plant materials and growth conditions.

$r h m l-R$ was obtained from the Maize Genetics Cooperative at the University of Illinois (Champaign-Urbana), stock refer- ence $601 \mathrm{H}$. Three additional isolates $(\operatorname{rhm} 1-A, \operatorname{rhml}-B$, and rhm 1-C) were obtained by screening seedlings for $B$. maydis resistance in a primarily A632 genetic background from Robertson Mutator transposon-enriched populations. Allelism tests were performed by intercrossing each allele or by backcrossing $\mathrm{F}_{1}$ heterozygotes of each allele to $\mathrm{rhml}$ - $\mathrm{R}$ to generate 1:1 segregating progeny. For the experiments described below, rhml to wild-type comparisons were made either by comparing pooled $\mathrm{rhml}$ with pooled wild type from an $\mathrm{F}_{2}$ population (bulk segregant analysis) or by comparing rhml homozygous alleles with their respective heterozygotes from A632 or B73 outcrosses.

\section{Bipolaris maydis inoculation and assays.}

Plant growth conditions, the $B$. maydis source and inoculation methods, and the methods for assaying viable $B$. maydis in lesions were performed as described (Simmons et al. 1998). The number of lesions assayed for viable B. maydis propagules ranged from 84 to 145 per genotype, averaging 123. Resistance to B. maydis was scored 3 days postinoculation (dpi). At $1 \mathrm{dpi}$, wild type and $\mathrm{rhml}$ had visually indistinguishable mottled leaves resulting from the nascent lesions. To determine B. maydis sporulation in rhml and wild-type lesions, infected leaves at 3 dpi were surface sterilized in $70 \%$ ethanol for $3 \mathrm{~min}$, washed three times with sterile water, blotted dry, and placed in a humid chamber at ambient temperature for $40 \mathrm{~h}$, after which visual observations were made.

\section{PR protein analyses.}

The PR1 and chitinase Western blot analysis was performed as described (Simmons et al. 1998). For the peroxidase isozyme activity assay, $100 \mu \mathrm{g}$ of ground frozen powder for each sample was extracted in $1 \mathrm{ml}$ of $\mathrm{pH} 7.4,0.1 \mathrm{M}$ sodium phosphate buffer, followed by centrifugation at $10,000 \mathrm{~g}$ for $15 \mathrm{~min}$. The supernatants $(15 \mu \mathrm{l})$ for these samples were subjected to isoelectric focusing electrophoresis with precast, wide-range ( $\mathrm{pH} 3.5$ to 9.5) polyacrylamide gels (Amersham Pharmacia Biotech, Piscataway, NJ, U.S.A.), followed by peroxidase activity visualization, essentially as described (Dowd 1994).

\section{Differential display mRNA profiling.}

Profiling mRNA levels in rhml and wild-type plants before and after inoculation was performed with GeneCalling technology (Bruce et al. 2000; Shimkets et al. 1999). This technology involves a genome-wide, high-throughput quantitative mRNA differential display of PCR-amplified restriction enzyme-digested cDNA fragments that were size separated by slab gel or capillary electrophoresis. Gene identities can be determined by comparing the patterns of coordinately expressed cDNA fragments with computer-generated virtual restriction enzyme digests of cDNA sequence datasets, which yields a GeneCall score. This score is related to the number of observed coordinately expressed cDNA fragments for a gene relative to the expected number of cDNA fragments for the gene. At the time of this study, the sequence database involved approximately 200,000 maize-expressed cDNA ESTs (proprietary to Pioneer Hi-Bred International), which were supplemented with then-available public maize cDNA sequences from GenBank. Gene identities were confirmed by a competitive PCR reaction that involved a reamplification of the sam- 
ple in the presence of unlabeled oligonucleotide primers designed from the candidate gene sequence, although 10 nucleotides internal from the original restriction sites were used to identify the cDNA fragment in question. If the gene is identified correctly, then the cDNA fragment in the competitive PCR reaction is not labeled and thus appears absent and, consequently, the gene is confirmed.

Three $r h m l$ CuraGen experiments were performed, two involving $r h m l-C$ and a third $r h m l-B$. For each, the $\mathrm{F}_{2}$ population was planted in two sets, one to be inoculated with a control $0.02 \%$ aqueous Tween 20 solution, the other with $B$. maydis spores. Similar portions of L4, L5, and L6 leaf tissue were collected and frozen $24 \mathrm{~h}$ postinoculation. Individual plants were scored for the $r h m 1$ phenotype $96 \mathrm{~h}$ postinoculation, after which the frozen $r h m 1$ or wild-type tissues, control or inoculated, from at least six rhml plants and up to 18 wildtype plants, were then pooled separately. Total RNA from each pool was isolated from $4 \mathrm{~g}$ of leaf powder by the TriReagent method (Molecular Research Center, Cincinnati, $\mathrm{OH}$, U.S.A.) and sent to CuraGen for analysis. The results were evaluated and analyzed with CuraGen GeneScape software.

\section{Rhm1 and hydroxamic acid phenotypes in $r h m 1-b x 1$ double mutants.}

The $b x l$ gene encodes a tryptophan synthase alpha homolog that forms indole, the precursor for the synthesis of all hydroxamic acids, including DIMBOA (Frey et al. 1997). A bxl mutant (courtesy of D. Weber, Illinois State University) was crossed separately to $r h m l-\mathrm{B}$ and $r h m l-\mathrm{C}$, and the $\mathrm{F}_{1}$ were selfed. From a representative ear of each of these $\mathrm{F}_{2}$ populations, 220 seeds were planted. Controls of rhml-B and rhml$\mathrm{C}$ and $b x l$ also were planted, 10 seeds each. The $0.8-\mathrm{cm}$ tips of dark-grown germinating seedling coleoptiles $(3 \mathrm{~cm}$ above greenhouse soil on day 4) were excised, crushed slightly with a glass rod, and submerged in $\mathrm{FeCl}_{3}$-saturated $0.12 \mathrm{~N} \mathrm{HCl}$ in $94 \%$ ethanol to stain for hydroxamic acids. The wild-type $B x$ tissues stained brown-black, whereas mutant $b x$ tissues remained clear. The seedlings were then transferred to the light and on day 14 were inoculated with $B$. maydis. The rhml phenotypes were first recorded on day 18 , followed immediately by reinoculation. The plants were scored again on days 21 through 24 for confirmation. Plants failing to germinate or those of an uncertain phenotype were omitted from further genetic segregation analysis.

\section{ACKNOWLEDGMENTS}

We thank S. Briggs, K. Bruce, D. Christensen, B. Dolezal, J. Duvick, B. Fleener, C. Jensen, L. Marshall, P. Navarro, T. Nevshemal, K. Searcy, K. Simcox, V. Sondag, J. Thompson, J. Tossberg, D. Wilkinson, and M. Yates for their assistance and helpful comments.

\section{LITERATURE CITED}

Agrios, G. N. 1997. Plant Pathology, 4th ed. Academic Press, San Diego, CA, U.S.A.

Babcock, G. D., and Esen, A 1994. Substrate specificity of maize $\beta$ glucosidase. Plant Sci. 101:31-39.

Bravo, H. R., and Niemeyer, H. M. 1991. Decomposition of 7-nitro-2,4dihydroxy-1,4-benzoxazin-3-one in aqueous solutions. Heterocycles 32:1687-1691.

Bruce, W., Folkerts, O., Garnaat, C., Crasta, O., Roth, B., and Bowen, B. 2000. Expression profiling of the maize flavonoid pathway genes con- trolled by estradiol-inducible transcription factors CRC and P. Plant Cell 12:65-80.

Burnette, D. C., and White, D. G. 1985. Inheritance of resistance to Bipolaris maydis race $\mathrm{O}$ in crosses derived from nine resistant inbred lines of maize. Phytopathology 75:1195-1200.

Buschges, R., Hollricher, K., Panstruga, R., Simons, G., Wolter, M., Frijters, A., Van-Daelen, R., Van-Der-Lee, T., Diergarde, P., Groenendijk, J., Topsch, S., Vos, P., Salamini, F., and Schulze-Lefert, P. 1997. The barley Mlo gene: A novel control element of plant pathogen resistance. Cell 88:695-705.

Chen, W. P., Chen, P. D., Liu, D. J., Kynast, R., Friebe, B., Velazhahan, R., Muthukrishnan, S., and Gill, B. S. 1999. Development of wheat scab symptoms is delayed in transgenic wheat plants that constitutively express a rice thaumatin-like protein gene. Theor. Appl. Genet. 99:755-760.

Clarke, J. D., Liu, Y., Klessig, D. F., and Dong, X. 1998. Uncoupling PR gene expression from NPR1 and bacterial resistance: Characterization of the dominant Arabidopsis cpr6-1 mutant. Plant Cell 10:557-569.

Conn, E. E. 1993. Beta-glycosidases in plants-substrate specificity. Pages 15-26 in: Beta-Glucosidases: Biochemistry and Molecular Biology. Am. Chem. Soc. Symp. Ser., Vol. 533. A. Esen, ed. American Chemical Society, Washington, DC.

Craig, J., and Fajemisin, J. M. 1969. Inheritance of chlorotic lesion resistance to Helminthosporium maydis in maize. Plant Dis. Rep. 53:742-743.

Cuevas, L., Neimeyer, H. M., and Jonsson, L. M. V. 1992. Partial purification and characterization of a hydroxamic acid glucoside- $\beta$-Dglucosidase from maize. Phytochemistry 31:2609-2612.

Cutt, J. R., and Klessig, D. F. 1992. Pathogenesis-related proteins. Pages 209-243 in: Genes Involved in Plant Defense. T. Boller and F. Meins, eds. Springer-Verlag, Vienna.

Dowd, P. F. 1994. Enhanced maize (Zea mays L.) pericarp browning: Associations with insect resistance and involvement of oxidizing enzymes. J. Chem. Ecol. 20:2777-2803.

Esen, A. 1993. Beta-glucosidases-Overview. Pages 1-14 in: BetaGlucosidases: Biochemistry and Molecular Biology. Amer. Chem. Soc. Symp. Ser., Vol. 533. A. Esen, ed. American Chemical Society, Washington, DC.

Esen, A., and Stetler, D. A. 1993. Subcellular localization of maize $\beta$ glucosidase. Maize Genet. Coop. Newsl. 67:19-20.

Esnault, R., Buffard, D., Breda, C., Sallaud, C., El Turk, J., and Kondorosi, A. 1993. Pathological and molecular characterizations of alfalfa interactions with compatible and incompatible bacteria, Xanthomonas campestris pv. alfalfae and Pseudomonas syringae pv. pisi. Mol. Plant-Microbe Interact. 6:655-664.

Feldwisch, J., Vente, A., Zettl, R., Bako, L., Campos, N., and Palme, K. 1994. Characterization of two membrane-associated $\beta$-glucosidases from maize (Zea mays L.) coleoptiles. Biochemistry 302:15-21.

Frey, M., Chomet, P., Glawischnig, E., Stettner, C., Grün, S., Winklmair, A., Eisenreich, W., Bacher, A., Meeley, R. B., Briggs, S. P., Simcox, K., and Gierl, A. 1997. Analysis of a chemical plant defense mechanism in grasses. Science 277:696-699.

Holley, R. N., and Goodman, M. M. 1989. New sources of resistance to Southern corn leaf blight from tropical hybrid maize derivatives. Plant Dis. 73:562-564.

Hughes, M. A. 1993. Molecular genetics of plant cyanogenic betaglucosidases. Pages 153-169 in: Biochemistry and Molecular Biology. Amer. Chem. Soc. Symp. Ser., Vol. 533. A. Esen, ed. American Chemical Society, Washington, DC.

Linthorst, H. J. M. 1991. Pathogenesis-related proteins of plants. Crit. Rev. Plant Sci. 10:123-150.

Neuffer, M. G., Coe, E. H., and Wessler, S. R. 1997. Mutants of Maize. Cold Spring Harbor Laboratory, Cold Spring Harbor, NY, U.S.A.

Niemeyer, H. M. 1988. Hydroxamic acids (4-hydroxy-1,4-benzoxazin-3ones), defense chemicals in the Gramineae. Phytochemistry 27:33493358.

Nishizawa, Y., Nishio, Z., Nakazono, K., Soma, M., Nakajima, E., Ugaki, M., and Hibi, T. 1999. Enhanced resistance to blast (Magnaporthe grisea) in transgenic Japonica rice by constitutive expression of rice chitinase. Theor. Appl. Genet. 99:383-390.

Osbourn, A. E. 1996. Preformed antimicrobial compounds and plant defense against fungal attack. Plant Cell 8:1821-1831.

Peterhaensel, C., Freialdenhoven, A., Kurth, J., Kolsch, R., and SchulzeLefert, P. 1997. Interaction analyses of genes required for resistance 
responses to powdery mildew in barley reveal distinct pathways leading to leaf cell death. Plant Cell 9:1397-1409.

Pryor, A. J. 1978. Mapping of glucosidase on chromosome 10. Maize Genet. Coop. Newsl. 52:14.

Queirolo, C. B., Andreo, C. S., Vallejos, R. H., Niemeyer, H. M., and Corcuera, L. J. 1981. Effects of hydroxamic acids isolated from Gramineae on adenosine $5^{\prime}$-triphosphate synthesis in chloroplasts. Plant Physiol. 68:941-943.

Reimers, P. J., Guo, A., and Leach, J. E. 1992. Increased activity of a cationic peroxidase associated with an incompatible interaction between Xanthomonas oryzae pv oryzae and rice (Oryza sativa). Plant Physiol. 99:1044-1050.

Sharma, S. G., Narayan, R., Lal, S., and Chaturvedi, C. 1983. Role of phenolic compounds in resistance of maize to leaf blight caused by Dreschslera state of Cochliobolus heterostrophus. Indian J. Phytopathol. 36:43-46.

Shimkets, R. A., Lowe, D. G., Tai, J. T., Sehl, P., Jin, H., Yang, R., Predki, P. F., Rothberg, B. E., Murtha, M. T., Roth, M. E., Shenoy, S. G., Windemuth, A., Simpson, J. W., Simons, J. F., Daley, M. P., Gold, S. A., McKenna, M. P., Hillan, K., Went, G. T., and Rothberg, J. M. 1999. Gene expression analysis by transcript profiling coupled to a gene database query. Nature Biotechnol. 17:798-803.

Simmons, C., Hantke, S , Grant, S., Johal, G. S., and Briggs, S. P. 1998 The maize lethal leaf spot 1 mutant has elevated resistance to fungal infection at the leaf epidermis. Mol. Plant-Microbe Interact. 11:11101118.

Smith, D. R., and Hooker, A. L. 1973. Monogenic chlorotic-lesion resistance in corn to Helminthosporium maydis. Crop Sci. 13:330-331.

Smith, D. R., and White, D. G. 1988. Diseases of corn. Pages 687-766 in: Corn and Corn Improvement, 3rd ed. F. F. Sprague and J. W. Dudley, eds. American Society of Agronomy-Crop Science Society of America-Soil Science Society of America Publishers, Madison, WI, U.S.A.

Vad, K., De-Neergaard, E., Madriz-Ordenana, K., Mikkelsen, J. D., Collinge, D. 1993. Accumulation of defence-related transcripts and cloning of a chitinase mRNA from pea leaves (Pisum sativum L.) inoculated with Ascochyta pisi Lib. Plant Sci. 92:69-79.

White, D. G. 1999. Compendium of Corn Diseases, 3rd ed. American Phytopathological Society Press, St. Paul, MN, U.S.A.

Zaitlin, D., DeMars, S., and Ma, Y. 1993. Linkage of rhm, a recessive gene for resistance to southern corn leaf blight, to RFLP marker loci in maize (Zea mays) seedlings. Genome 36:555-564. 